\title{
A pursuit game with range-only measurements
}

\author{
Shaunak D. Bopardikar
}

Francesco Bullo

João P. Hespanha

\begin{abstract}
We address a discrete-time, pursuit-evasion game with alternate moves played between two kinds of players: the pursuer and the evader. The pursuer wishes to capture the evader while the evader's goal is to avoid capture. By capture, we mean that the distance between the players is no greater than 1 unit. We assume simple, first-order motion kinematics for the players. The pursuer can move with a step size of at most 1 unit while the evader can move with a maximum step size of $\beta<1$ units. The pursuer is able to measure only its distance from the evader, before and after the evader's move. We propose a capture strategy and first show that for the game played in $\mathbb{R}^{2}$, if $\beta<0.5$, then a single pursuer captures the evader in finite time. Next, we show that if the game is played in $\mathbb{R}^{3}$ and if $\beta<0.5$, then with a modified strategy, two identical cooperative pursuers capture the evader in finite time. Finally, we shed light on the performance of the capture strategy in the case of $\beta \in[0.5,1[$ and the case of sensing errors via simulations. We also present a simulation study of a version of this game with simultaneous moves.
\end{abstract}

\section{INTRODUCTION}

The game of pursuit can be posed as to determine a strategy for a pursuer to capture an evader in a given environment. By capture, we mean that the evader and the pursuer are within a specified distance after a finite time. The aim of the pursuer is to capture the evader for any evader strategy. The evader wins the game if it can avoid capture indefinitely. Capture strategies are important in surveillance where the goal is to detect and capture intruders that move unpredictably. Another application is search-and-rescue operations where a worst-case capture strategy guarantees a rescue, in spite of any unpredictable motion of the victim.

\section{A. Related Work}

There has been tremendous interest in pursuit-evasion games ever since their formal introduction [1]. Various versions of these games have been studied over the past four decades - for instance [2], [3] and [4] to cite a few. Recently, there has been a surge of interest in the algorithmic approach to the game in discrete-time. [5] gives sufficient conditions and a strategy for a single pursuer to capture an evader in a semiopen environment. [6] and [7] analyze pursuer strategies of moving towards the current and towards the last position of the evader respectively. [8] and [9] address visibility-based pursuit evasion. With respect to multiple cooperative pursuers, [10] addresses capturing an equally fast evader in a boundaryless

This material is based upon work supported in part by ARO MURI Award W911NF-05-1-0219, ONR Award N00014-07-1-0721 and by the Institute for Collaborative Biotechnologies through the grant DAAD19-03-D-0004 from the U.S. Army Research Office.

Shaunak D. Bopardikar, Francesco Bullo and João P. Hespanha are with the Center for Control, Dynamical Systems and Computation (CCDC), University of California at Santa Barbara, Santa Barbara, CA 93106, USA, \{shaunak, bullo\}eengineering.ucsb.edu, hespanha@ece.ucsb.edu environment while [11] deals with locating and capture in polygonal environments.

With respect to pursuit under sensing constraints, [12] deals with a version of pursuer's visibility limited to a cone. [13] considers a graph environment, with the visibility limited to adjacent nodes, while [14] and [15] propose a framework assuming probabilistic models for sensing. [16] addresses the case in which the pursuer only knows an approximate location of the evader. [17] and [18] present a solution to the game under bounded measurement uncertainty in sensing the evader.

Other areas of research related to the problem we address, are target tracking and localization. Using distance-only measurements, [19] determines optimal motions for multiple mobile sensors to minimize the error in the posterior estimate of the target position. Using the Fisher Information Matrix, [20] characterizes a condition for local system observability of tracking a moving target in a plane with range-only measurements. [21] and [22] present an established estimation method to track targets moving with bounded speeds. We refer to this method as the Grow-Intersect algorithm.

\section{B. Contributions}

We address a discrete-time alternate-motion pursuit-evasion game played between two kinds of players, the pursuer and the evader. The pursuer wishes to capture the evader while the evader's goal is to avoid capture. By capture, we mean that the distance between the pursuer and the evader is no greater than 1 unit. The game is played in $\mathbb{R}^{2}$, i.e., the unbounded plane. We assume simple, first-order motion kinematics for both players. The pursuer can move with a step size of at most 1 unit while the evader can move with a maximum step size of $\beta<1$. The pursuer is able to measure its distance from the evader before as well as after the evader's move, while the evader is assumed to have complete information of the pursuer's location. In continuous time, this is analogous to the pursuer being equipped with a sensor that measures the distance to the evader as well as the rate of change of this distance. [23] presents an example of one such sensor.

In the proposed game, we present a strategy inspired by the Grow-Intersect algorithm for the pursuer and show that: (i) if the maximum evader step size $\beta<0.5$, then the pursuer captures the evader in finite time, (ii) for the game played in $\mathbb{R}^{3}$ : if $\beta<0.5$, then two identical, cooperative pursuers capture the evader in finite time, and (iii) we provide upper bounds on the time taken to capture the evader in parts (i) and (ii). Finally, we present simulation studies in the planar case to address: (i) the case of $\beta \in[0.5,1$ [, (ii) the effect of additive, zero-mean Gaussian noise with variance proportional to the square of the distance between the evader and the pursuer on the outcome of the game, and (iii) a game with simultaneous moves. 


\section{Organization}

The problem formulation is described in Section II. The capture strategy and main result is presented in Section III. A cooperative pursuit version of this game is presented in Section IV. Due to space constraints, the proofs of the main results in Sections III and IV are provided in our online technical report [24]. Simulations that address the case of evader speed $\beta \in[0.5,1[$ and sensor noise are presented in Section V. A version of the present game with simultaneous moves and a simulation study of the application of a modified capture strategy are presented in Section VI.

\section{Problem Set-uP}

We assume a discrete-time model with alternate motion of the evader and the pursuer. The game is played in the unbounded plane. We assume simple, first-order motion kinematics for both players. The pursuer can move with a step size of at most 1 unit while the evader can move with a step size of $\beta<1$. The pursuer is equipped with a range-only sensor that measures its distance from the evader. The evader is assumed to know exact information of the pursuer's location. Further, we assume that at each time instant, the players take measurements before and after the pursuer's move. Thus a sequence of the game consists of the following: (i) the evader moves, (ii) players take measurements, (iii) the pursuer moves, (iv) the players take measurements. This is shown in Figure 1. Capture is defined when the evader is not greater than a unit distance from the pursuer.

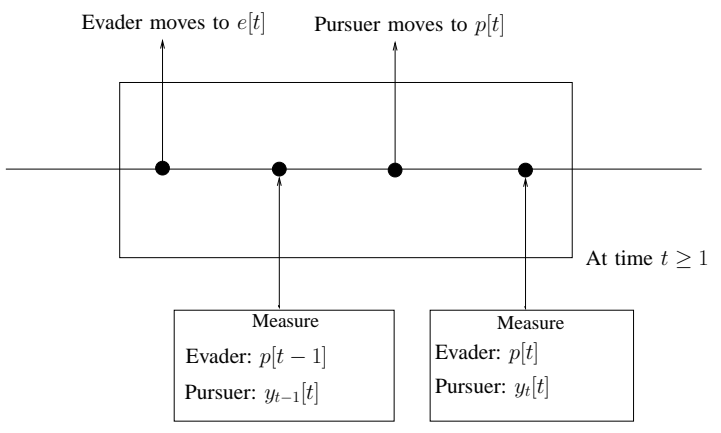

Fig. 1. A sequence at each time instant $t \in\{1,2, \ldots\}$ in our alternate motion model. The players take measurements before and after the pursuer's move.

Let $e[t] \in \mathbb{R}^{2}$ and $p[t] \in \mathbb{R}^{2}$ denote the positions of the evader and the pursuer respectively, at time $t \in \mathbb{Z}_{\geq 1}$. The discrete-time equations of motion are

$$
\begin{aligned}
& e[t]=e[t-1]+u^{e}\left(e[t-1],\{p[\tau]\}_{\tau=0}^{t-1}\right), \\
& p[t]=p[t-1]+u^{p}\left(p[t-1], y_{t-1}[t-1], y_{t-1}[t]\right),
\end{aligned}
$$

where $\{p[\tau]\}_{\tau=1}^{t-1}$ denotes the set $\{p[0], p[1], \ldots, p[t-1]\}$. For the pursuer, at the $t^{t h}$ time instant , $y_{t-1}[t-1], y_{t-1}[t] \in$ $\mathbb{R}_{\geq 0}$ are the distances of the evader's position from the pursuer before and after the pursuer's move respectively. Thus, $y_{t-1}[t-1]=\|e[t-1]-p[t-1]\|$ and $y_{t-1}[t]=\|e[t]-p[t-1]\|$. The functions $u^{e}: \mathbb{R}^{2} \times \underbrace{\mathbb{R}^{2} \times \cdots \times \mathbb{R}^{2}}_{t \text { times }} \rightarrow \mathbb{R}^{2}$ and $u^{p}:$
$\mathbb{R}^{2} \times \mathbb{R} \times \mathbb{R} \rightarrow \mathbb{R}^{2}$ are termed as strategies for the evader and pursuer respectively. Notice that in this formulation, we allow the evader the access to the entire history of the pursuer's motion, while we allow the pursuer the access to only two of the most recent evader measurements. The lack of symmetry between the number of arguments in strategies of the evader and the pursuer is due to the alternate motion model and due to the assumptions on the measurement models of the players.

Since the step sizes of each player are bounded, we have

$$
\left\|u^{e}\right\| \leq \beta, \quad \text { and } \quad\left\|u^{p}\right\| \leq 1,
$$

where $\beta<1$. Capture takes place when for some $T_{\text {cap }} \in \mathbb{Z}_{\geq 0}$,

$$
\left\|e\left[T_{\text {cap }}\right]-p\left[T_{\text {cap }}-1\right]\right\| \leq 1 \quad \text { or } \quad\left\|e\left[T_{\text {cap }}\right]-p\left[T_{\text {cap }}\right]\right\| \leq 1 .
$$

The problem is to determine a pursuer strategy $u^{p}$ that guarantees capture for any evader strategy $u^{e}$.

Remark II.1 (Continuous-time analogy) Such a model arises when one discretizes the continuous time pursuitevasion game in which the pursuer is equipped with a sensor that continuously measures the distance to the evader as well as the rate of change of this distance.

\section{The Capture Strategy and Main Result}

In this section, we describe our capture strategy and the corresponding main result.

Our capture strategy has two phases: Initialization and Pursuit. These are described as follows.

\section{A. Initialization phase}

This phase lasts for only the first sequence. In the first sequence,

(i) The evader moves to $e[1]$.

(ii) The pursuer gets the measurement $y_{0}[1]$ and it constructs $\partial \mathcal{B}_{y_{0}[1]}(p[0])$ which is a circle of radius $y_{0}[1]$ around the point $p[0]$.

(iii) The pursuer randomly selects a direction to move and moves along it with unit step size.

(iv) The pursuer gets the measurement $y_{1}[1]$ and it constructs $\partial \mathcal{B}_{y_{1}[1]}(p[1])$ and computes the estimate

$$
\hat{E}[1]:=\partial \mathcal{B}_{y_{1}[1]}(p[1]) \cap \partial \mathcal{B}_{y_{0}[1]}(p[0]) .
$$

Since $\hat{E}[1]$ is an intersection of two non-concentric circles described in the right hand side of (4), $\hat{E}[1]=\left(\hat{e}_{a}[1], \hat{e}_{b}[1]\right) \in$ $\mathbb{R}^{2} \times \mathbb{R}^{2}$ is an estimate of $e[1]$. If $\hat{e}_{a}[1]=\hat{e}_{b}[1]$, then the pursuer has accurately determined $e[1]$. In general, the pursuer is unable to distinguish between the two estimates.

1) Pursuit phase: We now present our pursuit strategy.

Until the evader is not captured, at time $t \geq 2$,

(i) the pursuer selects a point $\hat{e}[t-1] \in \hat{E}[t-1]$ at random and moves towards it with full step size. Thus,

$$
p[t]=p[t-1]+\frac{\hat{e}[t-1]-p[t-1]}{\|\hat{e}[t-1]-p[t-1]\|} .
$$


(ii) The pursuer updates the estimate of the evader's position using

$$
\begin{aligned}
& \hat{E}[t]:=\partial \mathcal{B}_{y_{t-1}[t]}(p[t-1]) \cap \\
&\left(\hat{E}[t-1] \oplus \mathcal{B}_{\beta}(0)\right) \cap \partial \mathcal{B}_{y_{t}[t]}(p[t]),
\end{aligned}
$$

where $\mathcal{B}_{\beta}(0) \subset \mathbb{R}^{2}$ denotes the closed circular region of radius $\beta$ around the origin $0 \in \mathbb{R}^{2}$ and the operation $\oplus$ denotes the Minkowski sum in the plane.

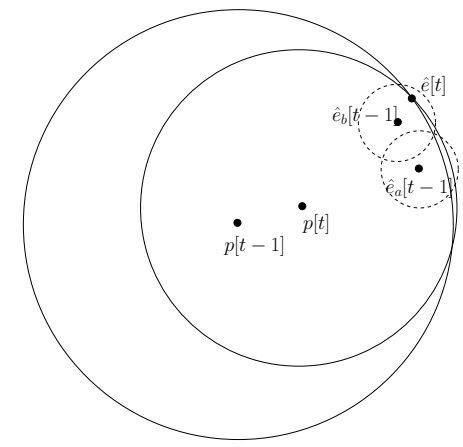

Fig. 2. An instance of the pursuit strategy. The dotted circles have radii equal to $\beta$ and denote the region where the evader can step at time $t$. This figure illustrates the case when the pursuer moves towards $\hat{e}_{a}[t-1]$ while the evader was actually at $\hat{e}_{b}[t-1]$ and consequently exactly localizes the evader at time $t$.

An instance of this strategy is shown in Figure 2. A simple induction argument gives the following result.

Lemma III.1 (Evader estimate) At every time instant $t \in$ $\mathbb{Z}_{\geq 1}$,

(i) The evader's position $e[t] \in \hat{E}[t]$, where $\hat{E}[t]$ is recursively defined using (4) and (6).

(ii) The set $\hat{E}[t]$ contains at most two points $\left(\hat{e}_{a}[t], \hat{e}_{b}[t]\right) \in$ $\mathbb{R}^{2} \times \mathbb{R}^{2}$. Further, $\left\|\hat{e}_{a}[t]-p[t]\right\|=\left\|\hat{e}_{b}[t]-p[t]\right\|$, for every $t$.

We now present the main result of this section.

Theorem III.2 (Capture in $\mathbb{R}^{2}$ ) If $\beta<0.5$, then a single pursuer captures the evader using our capture strategy and in at most $\left\lceil\frac{2(\|e[0]-p[0]\|+(1+2 \beta))}{(1-2 \beta)}\right\rceil$ time steps.

Remark III.3 (Single pursuer in $\mathbb{R}^{3}$ ) In $\mathbb{R}^{3}$, it is not clear whether it is possible to guarantee capture with a single pursuer using the proposed strategy. At each time instant $t$, the set of evader estimates $\hat{E}[t]$ in general contains more than just two points. This motivates the use of another cooperative pursuer in $\mathbb{R}^{3}$, which we address in the next section.

\section{Remark III.4 (Other Sensor-based formulations) The} Grow-Intersect algorithm can also be adapted to design a pursuit strategy when the evader is a transmitter device and the pursuer is equipped with a sensor that determines only the line that contains their positions, without the orientation sense. Our strategy guarantees that the pursuer simultaneously captures the evader as well as ascertains that it is within unit radius if the maximum evader speed $\beta<0.25$.

\section{Cooperative PURSUIT IN $\mathbb{R}^{3}$}

We now present the pursuit problem in $\mathbb{R}^{3}$ played with two cooperative pursuers.

\section{A. Problem statement and notation}

The problem formulation is almost identical to the planar case except that now we have two identical pursuers which move simultaneously at their turn. The game is played in $\mathbb{R}^{3}$. Akin to (1), the equations of motion are given by

$$
\begin{aligned}
e[t] & =e[t-1]+u^{e}\left(e[t-1],\{p[\tau]\}_{\tau=0}^{t-1}\right), \\
p_{i}[t] & =p_{i}[t-1]+u^{p_{i}}\left(p_{i}[t-1], y_{t-1}^{i}[t], y_{t}^{i}[t]\right),
\end{aligned}
$$

where for the $i^{t h}$ pursuer, $p_{i}[t] \in \mathbb{R}^{3}$ denotes its position at time $t, y_{t-1}^{i}[t], y_{t}^{i}[t] \in \mathbb{R}_{\geq 0}$ are the distances of the evader from it before and after the pursuers' move respectively and $u^{p_{i}}$ is its strategy. The strategies satisfy (2) and capture is defined when for some $i \in\{1,2\}$, (3) is satisfied.

The problem is to determine pursuer strategies $u^{p_{i}}$ that guarantee capture for any evader strategy $u^{e}$.

\section{B. Capture strategy and Main result}

We present our solution to the cooperative pursuit game played in $\mathbb{R}^{3}$. Again, our capture strategy has two phases: Initialization and Pursuit. These are described as follows.

1) Initialization phase: This phase lasts for only the first sequence. In the first sequence,

(i) The evader moves to $e[1]$.

(ii) For $i=\{1,2\}$, pursuer $p_{i}$ gets the measurement $y_{0}^{i}[1]$ and it constructs $\partial \mathcal{B}_{y_{0}^{i}[1]}\left(p_{i}[0]\right)$ which is the surface of a sphere of radius $y_{0}^{i}[1]$ around the point $p_{i}[0]$.

(iii) Pursuer $p_{i}$ selects a direction to move ensuring that $p_{1}[1] \neq p_{2}[1]$ and moves along it with unit step size.

(iv) Each pursuer $p_{i}$ gets the measurement $y_{1}^{i}[1]$ and it constructs $\partial \mathcal{B}_{y_{1}^{i}[1]}\left(p_{i}[1]\right)$ and computes the estimate

$$
\hat{E}[1]:=\bigcap_{i \in\{1,2\}}\left(\partial \mathcal{B}_{y_{1}^{i}[1]}\left(p_{i}[1]\right) \cap \partial \mathcal{B}_{y_{0}^{i}[1]}\left(p_{i}[0]\right)\right) .
$$

For each $i \in\{1,2\}$, the term in the outer parentheses in (7) is an intersection of the surfaces of two spheres in $\mathbb{R}^{3}$ and hence is a circle. Hence, $\hat{E}[1]$ is an intersection of two nonconcentric circles and thus contains at most two points.

2) Pursuit phase: We now present our pursuit strategy.

Until the evader is not captured, at time $t \geq 2$,

(i) If $\hat{E}[t-1]$ contains only one point $\hat{e}[t-1]$, then the pursuer closer to it, say $p_{1}$ moves towards it with full step size. The other pursuer $p_{2}$ moves:

a) towards $\hat{e}[t-1]$ with maximum step size, if the three points $\hat{e}[t-1], p_{1}[t-1]$ and $p_{2}[t-1]$ are not collinear.

b) anywhere inside except on the axis of a cone with half-angle equal to $\arcsin \left(\beta /\left\|e[t-1]-p_{2}[t-1]\right\|\right)$, vertex at $p_{2}[t-1]$ and with $e[t-1]-p_{2}[t-1]$ as the axis, with maximum step size, if the points $\hat{e}[t-1], p_{1}[t-1]$ and $p_{2}[t-1]$ are collinear. Refer to Figure 3 for an illustration.

In case both pursuers are equidistant, then pursuer $p_{1}$ is the one that moves directly towards the evader. 


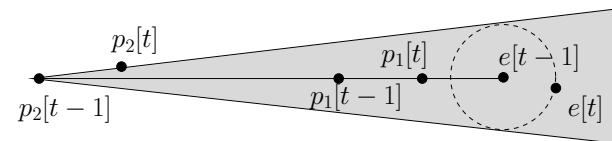

Fig. 3. The shaded region is the cone described in part (b) of item (i) of the Pursuit phase.

Otherwise, for $i=\{1,2\}$, each pursuer $p_{i}$ is assigned a unique point $\hat{e}_{i}[t-1]$ in $\hat{E}[t-1]$ and it moves towards it with full step size. Thus,

$$
p_{i}[t]=p_{i}[t-1]+\frac{\hat{e}_{i}[t-1]-p_{i}[t-1]}{\left\|\hat{e}_{i}[t-1]-p_{i}[t-1]\right\|} .
$$

(ii) The pursuer updates the estimate of the evader's position using

$$
\begin{array}{r}
\hat{E}[t]:=\left(\hat{E}[t-1] \oplus \mathcal{B}_{\beta}(0)\right) \bigcap_{i \in\{1,2\}}\left(\partial \mathcal{B}_{y_{t}^{i}[t]}\left(p_{i}[t]\right) \cap\right. \\
\left.\quad \partial \mathcal{B}_{y_{t-1}^{i}[t]}\left(p_{i}[t-1]\right)\right) .
\end{array}
$$

where $\mathcal{B}_{\beta}(0) \subset \mathbb{R}^{3}$ denotes the closed sphere of radius $\beta$ around the origin $0 \in \mathbb{R}^{3}$ and the operation $\oplus$ denotes the Minkowski sum in $\mathbb{R}^{3}$.

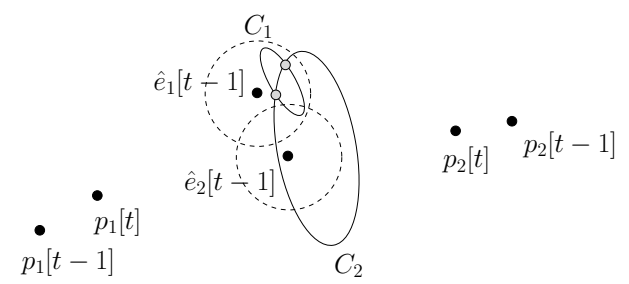

Fig. 4. An instance of the cooperative pursuit in $\mathbb{R}^{3}$. The dotted circles have radii equal to $\beta$ and denote the region where the evader can step at time $t$. Circles $C_{1}$ and $C_{2}$ (shown as ellipses here) are the intersections of the two spheres (not shown to preserve clarity) associated with each measurement for each pursuer. The lightly shaded dots is the set $\hat{E}[t]$.

An instance of this strategy is shown in Figure 4. Akin to Lemma III.1 in the single pursuer problem, we have the following result.

Lemma IV.1 (Evader estimate) At every time instant $t \in$ $\mathbb{Z}_{\geq 1}$,

(i) Using the proposed cooperative pursuit strategy, the two pursuers are at distinct locations in $\mathbb{R}^{3}$.

(ii) The set $\hat{E}[t]$ contains at most two points $\left(\hat{e}_{1}[t], \hat{e}_{1}[t]\right) \in$ $\mathbb{R}^{3} \times \mathbb{R}^{3}$. Further, for each $i \in\{1,2\},\left\|\hat{e}_{1}[t]-p_{i}[t]\right\|=$ $\left\|\hat{e}_{2}[t]-p_{i}[t]\right\|$, for every $t$.

(iii) The evader's position $e[t] \in \hat{E}[t]$, where $\hat{E}[t]$ is recursively defined using (7) and (9).

We now present the main result of this section.

Theorem IV.2 (Capture in $\mathbb{R}^{3}$ ) If $\beta<0.5$, then two pursuers capture the evader using the cooperative capture strategy and in at most $\left\lceil\frac{\left\|e[0]-p_{1}[0]\right\|+\left\|e[0]-p_{2}[0]\right\|+2(1+2 \beta)}{(1-2 \beta)}\right\rceil$ time steps.

\section{Simulation Studies}

We now present simulation studies that address the case of evader speed $\beta \in[0.5,1[$ and the case of the pursuer measurements being corrupted with additive, zero-mean Gaussian noise, with variance proportional to the square of the distance to the evader. All simulations were run using MATLAB ${ }^{\circledR}$.

\section{A. The case of $\beta \in[0.5,1[$}

We ran simulations for $\|e[0]-p[0]\|=20,30$ and 40 units. An upper limit of 1000 time steps was set to decide whether the capture strategy terminated into capture or evasion.

It is unclear as to what is the optimal evader strategy in this problem. This is because if the evader decides to always move directly away from the pursuer with full step (i.e., greedy move), then it would reduce the uncertainty in its position for the pursuer. If it does not make a greedy move, then the distance from the pursuer may reduce. So we adopt the following reasonable evader strategy for simulations - with full step, move to a point selected uniformly randomly in a sector with angle 0.2 radians. This sector is placed symmetrically along the line $e[t] p[t]$ and away from the pursuer.

The plots of probability of success of the strategy versus the evader speed $\beta$ are presented in Figure 5.

\section{B. Noisy measurements}

We now assume that the pursuer measurements are corrupted with zero-mean, additive Gaussian noise whose variance proportional to the square of the distance to the evader. This implies $\sigma_{t-1}[t]=\nu\|e[t]-p[t-1]\|$ and $\sigma_{t}[t]=$ $\nu\|e[t]-p[t]\|$, where $\nu>0$ is the noise parameter. Thus, in the notation of Section II, $y_{t-1}[t] \sim \mathcal{N}\left(\|e[t]-p[t-1]\|, \sigma_{t-1}[t]\right)$ and $y_{t}[t] \sim \mathcal{N}\left(\|e[t]-p[t]\|, \sigma_{t}[t]\right)$, where given $a, b \geq 0$, $\mathcal{N}(a, b)$ denotes the Gaussian distribution with mean $a$ and standard deviation $b$.

Since it is unclear as to what is the optimal evader strategy in this problem, we adopted the evader strategy in Section V-A. We ran simulations for $\beta=0.2,0.3$ and 0.4 units. The initial distance was set to 20 units. An upper limit of 2000 time steps was set to decide whether the capture strategy terminated into capture or evasion. The plots of probability of success of the strategy versus the noise parameter $\nu$ are presented in Figure 6.

\section{Vi. A Game with Simultaneous Moves: Simulation STUDY}

We now consider a discrete-time version of the game in the plane in which the pursuer and the evader move simultaneously. In this version, at each instant of time, each player gets only one measurement of its opponent. This is equivalent to a game in which the pursuer receives only the distance to the evader at each instant in continuous time. Thus, (1) becomes

$$
\begin{aligned}
& e[t]=e[t-1]+u^{e}\left(e[t-1],\{p[\tau]\}_{\tau=0}^{t-1}\right), \\
& p[t]=p[t-1]+u^{p}(p[t-1], y[t-1]) .
\end{aligned}
$$

We modify the capture strategy in Section III as follows. Initialization phase: The following happens simultaneously for only the first time step:

(i) The evader moves to $e[1]$. 


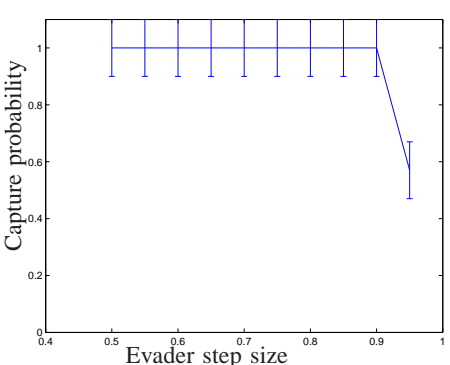

(a) Initial distance: 20 units

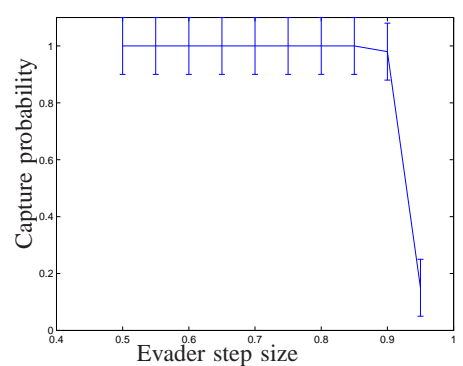

(b) Initial distance: 30 units

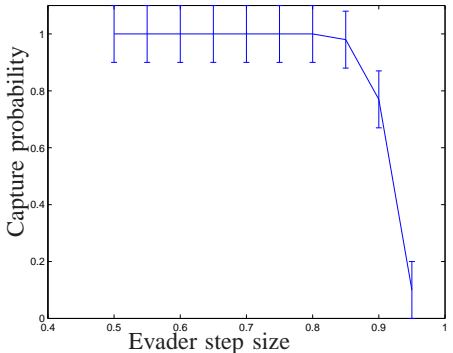

(c) Initial distance: 40 units

Fig. 5. Estimate of capture probability versus evader step size $\beta$. The vertical bars give a $95 \%$ confidence interval about the probability estimate $P(\beta)$ which is given by $\left[P(\beta)-2 \sqrt{\frac{0.25}{n}}, P(\beta)+2 \sqrt{\frac{0.25}{n}}\right.$, where $n=100$ is the number of trials [25]. For a particular evader strategy, we study how the capture strategy performs for evader step size $\beta \in[0.5,1[$.

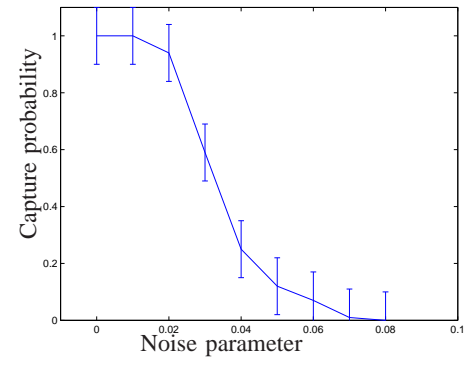

(a) Evader step size: $\beta=0.2$

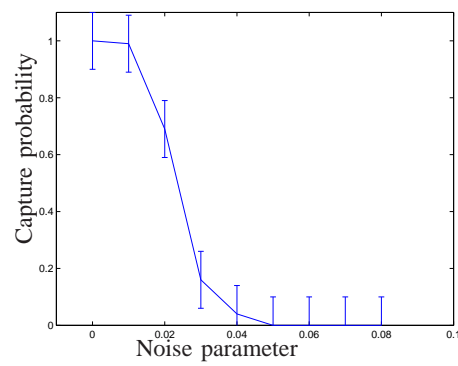

(b) Evader step size: $\beta=0.3$

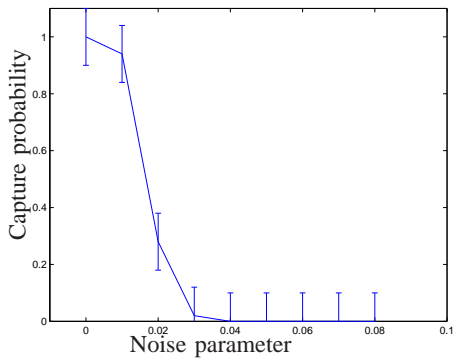

(c) Evader step size: $\beta=0.4$

Fig. 6. Estimate of capture probability versus noise parameter $\nu$. The vertical bars give a $95 \%$ confidence interval about the probability estimate $P(\nu)$ computed as described in Figure 5. For a particular evader strategy, we study how the capture strategy performs under noisy measurements.

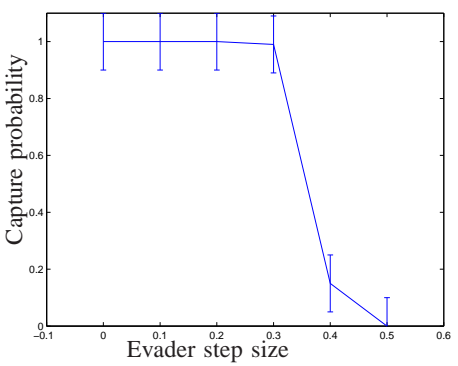

(a) Initial distance: 20 units

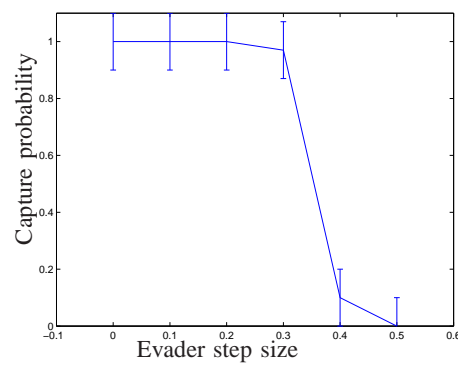

(b) Initial distance: 30 units

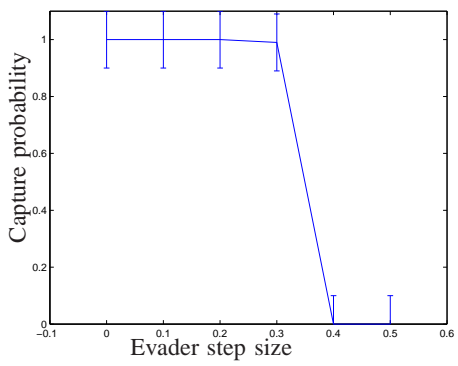

(c) Initial distance: 40 units

Fig. 7. Estimate of capture probability versus evader step size $\beta$, in the game with simultaneous moves. The vertical bars give a $95 \%$ confidence interval about the probability estimate $P(\beta)$, computed as described in Figure 5. For a particular evader strategy, we study the performance of a modified capture strategy presented in Section VI.

(ii) The pursuer randomly selects a direction to move and moves along it with unit step size.

(iii) The pursuer gets the measurement $y[1]$ and the evader estimate is given by

$$
\hat{E}[1]:=\partial \mathcal{B}_{y[1]}(p[1]) .
$$
2 ,

Pursuit Phase: Until the evader is not captured, at time $t \geq$

(i) If $\hat{E}[t-1]$ is a circle, then denote any point in it as $\hat{e}[t-1]$. Otherwise, denote as $\hat{e}[t-1]$ a point chosen uniformly randomly from one of the end points of the arcs in $\hat{E}[t-1]$. The pursuer moves towards $\hat{e}[t-1]$ with full step size.

(ii) The pursuer updates the estimate of the evader's position using

$$
\hat{E}[t]:=\left(\hat{E}[t-1] \oplus \mathcal{B}_{\beta}(0)\right) \cap \partial \mathcal{B}_{y[t]}(p[t]) .
$$

The strategy is illustrated in Figure 8. Since it is unclear as to what is the optimal evader strategy in this problem, we adopted the same evader strategy as in Section V-A. We ran simulations for $\|e[0]-p[0]\|=20,30$ and 40 units. An 


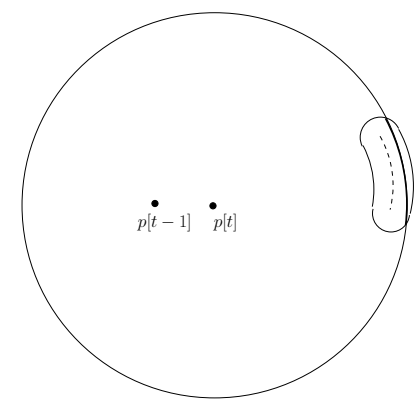

Fig. 8. Illustration of the pursuit strategy in the game with simultaneous moves. The dotted line is the estimate $\hat{E}[t-1]$. The bean-shaped region around it is its Minkowski sum with $\mathcal{B}_{\beta}(0)$ and the darkly shaded arc is the estimate $\hat{E}[t]$.

upper limit of 5000 time steps was set to decide whether the capture strategy terminated into capture or evasion. The plots of probability of success of the strategy versus the evader step size $\beta$ are presented in Figure 7.

\section{CONCLUSION AND Future DiRECTIONS}

We addressed a discrete-time alternate-motion pursuitevasion game in the plane in which the pursuer is equipped with a range-only sensor that measures its distance from the evader. We propose a capture strategy based on the established Grow-Intersect algorithm, and show that if the evader's maximum step size $\beta<0.5$, then the evader is captured. We then posed a variant of this game in $\mathbb{R}^{3}$ and showed that two cooperative pursuers capture the evader with a modified capture strategy if $\beta<0.5$. We gave upper bounds on the capture times in both games.

We then presented simulation studies that addressed the case of $\beta \in[0.5,1[$ and the case of noisy measurements in the planar game. We deduce that the proposed capture strategy performs fairly well against a reasonable evader strategy in the former case while in the latter case, we observe some amount of robustness to small values of the noise parameter. Finally, we presented a simulation study of a variant of this game with simultaneous moves. Based on simulation results, we deduce that the appropriately modified capture strategy succeeds with probability of more than $97 \%$ in the regime of $\beta \in[0,0.3]$.

In future, we plan to design a provably-correct capture strategy for the game with simultaneous moves. We also plan to shed more light on whether capture can be enforced with any arbitrarily small capture distance. With respect to alternate sensor formulations, we have been able to adapt the Grow-Intersect algorithm to design a pursuit strategy when the evader is a transmitter device and the pursuer is equipped with a sensor that determines only the line that contains their positions, without the orientation sense. Details of this casestudy will be a part of future work.

\section{REFERENCES}

[1] R. Isaacs, Differential games. New York: John Wiley, 1965.

[2] E. Cockayne, "Plane pursuit with curvature constraints," SIAM Journal on Control and Optimization, vol. 15, no. 6, pp. 1511-1516, 1967.

[3] J. Flynn, "Lion and Man: The general case," SIAM Journal on Control and Optimization, vol. 12, no. 4, pp. 581-597, 1974.
[4] T. D. Parsons, "Pursuit-evasion in a graph," in Theory and Applications of Graphs, ser. Lecture Notes in Mathematics. Springer Verlag, Nov. 1978, vol. 642, pp. 426-441.

[5] J. Sgall, "A solution of David Gale's lion and man problem," Theoretical Computational Science, vol. 259, no. (1-2), pp. 663-670, 2001.

[6] L. Alonso, A. S. Goldstein, and E. M. Reingold, "Lion and Man: Upper and lower bounds," ORSA Journal of Computing, vol. 4, no. 4, pp. 447452, 1992

[7] S. Alexander, R. Bishop, and R. Ghrist, "Pursuit and evasion in nonconvex domains of arbitrary dimension," in Robotics: Science and Systems II (Philadelphia PA), G. S. Sukhatme, S. Schaal, W. Burgard, and D. Fox, Eds. Cambridge, MA: MIT Press, 2007.

[8] L. J. Guibas, J.-C. Latombe, S. M. LaValle, D. Lin, and R. Motwani, "Visibility-based pursuit-evasion in a polygonal environment," in International Workshop on Algorithms and Data Structures, London, UK, 1997, pp. 17-30.

[9] S. Sachs, S. M. LaValle, and S. Rajko, "Visibility-based pursuit-evasion in an unknown planar environment," International Journal of Robotics Research, vol. 23, no. 1, pp. 3-26, 2004.

[10] S. Kopparty and C. V. Ravishankar, "A framework for pursuit evasion games in $\mathbb{R}^{n}$," Information Processing Letters, vol. 96, no. 3, pp. 114 122, 2005.

[11] V. Isler, S. Kannan, and S. Khanna, "Randomized pursuit-evasion in a polygonal environment," IEEE Transactions on Robotics, vol. 5, no. 21, pp. 875-884, 2005.

[12] B. P. Gerkey, S. Thrun, and G. Gordon, "Visibility-based pursuitevasion with limited field of view," in National Conference on Artificial Intelligence, San Jose, CA, Jul. 2004, pp. 20-27.

[13] V. Isler, S. Kannan, and S. Khanna, "Randomized pursuit-evasion with local visibility,' SIAM Journal on Discrete Mathematics, vol. 1, no. 20, pp. 26-41, 2006.

[14] J. P. Hespanha, H. J. Kim, and S. S. Sastry, "Multipleagent probabilistic pursuit-evasion games," Electrical Engineering and Computer Science, University of California at Berkeley, Tech. Rep., Aug. 1999, available electronically at http://www.ece.ucsb.edu/ hespanha/published.

[15] R. Vidal, O. Shakernia, H. Kim, D. H. Shim, and S. Sastry, "Probabilistic pursuit-evasion games: Theory, implementation and experimental evaluation," IEEE Transactions on Robotics and Automation, vol. 18, no. 2, pp. 662-669, 2002.

[16] G. Rote, "Pursuit-evasion with imprecise target location," in ACM-SIAM Symposium on Discrete Algorithms, Baltimore, MA, Jan. 2003, pp. 747753.

[17] B. N. Pshenichnyi, "Special pursuit and evasion problem with incomplete information," Cybernetics and Systems Analysis, vol. 31, no. 2, pp. 246-251, 1995.

[18] V. M. Kuntsevic and A. V. Kuntsevich, "Optimal control over the approach of conflicting moving objects under uncertainty," Cybernetics and Systems Analysis, vol. 38, no. 2, pp. 230-237, 2002.

[19] K. X. Zhou and S. I. Roumeliotis, "Optimal motion strategies for range-only distributed target tracking," in American Control Conference, Minneapolis, MN, Jun. 2006, pp. 5195-5200.

[20] T. L. Song, "Observability of target tracking with range-only measurements," IEEE Journal of Ocean Engineering, vol. 24, no. 3, pp. 383-387, 1999.

[21] F. C. Schweppe, "Recursive state estimation: Unknown but bounded errors and system inputs," IEEE Transactions on Automatic Control, vol. 13 , no. 1 , pp. 22-28, 1968.

[22] C. Detweiler, J. Leonard, D. Rus, and S. Teller, "Passive mobile robot localization within a fixed beacon field," in Workshop on Algorithmic Foundations of Robotics, New York, Jul. 2006.

[23] H. Ruser, A. V. Jena, V. Mágori, and H. R. Tränkler, "A low cost ultrasonic microwave multisensor for robust sensing of velocity and range," in Proceedings of Sensor '99, Nürnberg, May 1999.

[24] S. D. Bopardikar, F. Bullo, and J. P. Hespanha, "A pursuit game with range-only measurements," Center for Control, Dynamical Systems and Computation. University of California at Santa Barbara, Tech. Rep. CCDC-08-0906, 2008, available electronically at http: // ccdc.mee.ucsb. edu.

[25] R. E. Walpole, R. H. Myers, and S. L. Myers, Probability and Statistics for Engineers and Scientists, 6th ed. New Jersey, USA: Prentice Hall, 1998. 